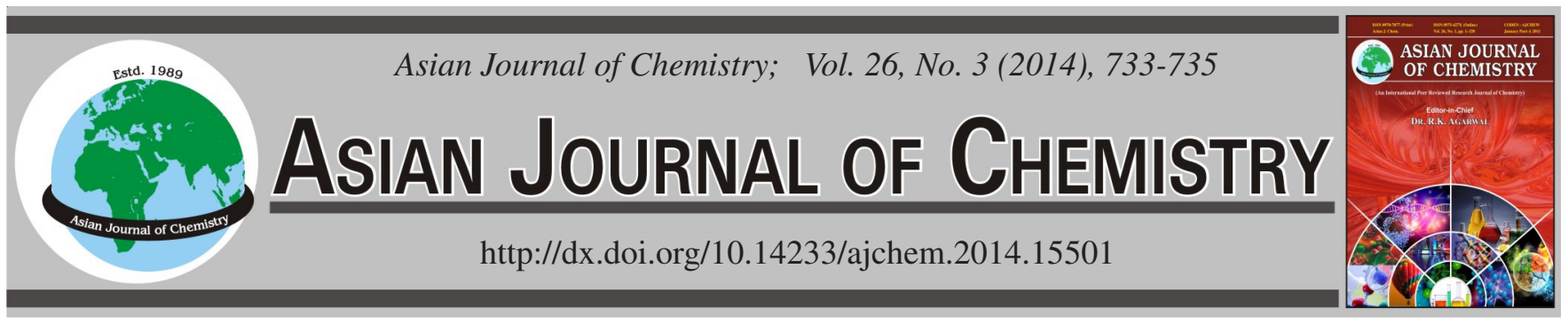

\title{
Synthesis and Structures of Mononuclear Co(III) and Cu(II) Complexes Derived from $\mathrm{N}$-[2-(2-Hydroxy-3-methoxybenzylideneamino)cyclohexyl]-2-hydroxybenzamide
}

\author{
Hui-Yu Wang, Yun-Hui Li, Yu-Qin Ma, Ying Gao, Gui-Miao Yu and Xiu-Yun Yang*
}

School of Chemistry and Environmental Engineering, Changchun University of Science and Technology, Changchun 130022, P.R. China

*Corresponding author: Fax: +86 0431 84727678; Tel: +860431 85582361; E-mail: liyh@ cust.edu.cn; whyu@ciac.jl.cn

Two mononuclear complexes, $\left[\mathrm{Co}(\mathrm{HL})_{2}\left(\mathrm{C}_{8} \mathrm{H}_{7} \mathrm{O}_{3}\right)\right] \cdot\left(\mathrm{CH}_{3} \mathrm{OH}\right)_{2}(\mathbf{1})$ and $\left[\mathrm{Cu}(\mathrm{HL})_{2}\right](\mathbf{2})$ where $\mathrm{H}_{2} L=\mathrm{N}$-[2-(2-hydroxy-3-methoxybenzylideneamino)cyclohexyl]-2-hydroxybenzamide have been synthesized and characterized by IR, elemental analysis and crystal structure analysis. In complexes (1) and (2) the core ion has the six-coordinated $\mathrm{N}_{2} \mathrm{O}_{4}$ environment with a distorted octahedral geometry and four-coordinated $\mathrm{N}_{2} \mathrm{O}_{2}$ environment with a distorted square-planar geometry, respectively.

Keywords: Cobalt(II), Copper(II), Complexes, Crystal structure.

\section{INTRODUCTION}

The novel transition-metal complexes with oxygen and nitrogen donor Schiff bases have received attention not only because of their fascinating self-assembled structures but also because of their important catalytic, magnetic and biological properties $^{1-3} . o$-Vanillin has allowed the construction of polymers with defined geometry and special properties; ${ }^{4,5}$ and the application of transition-metal, ${ }^{6,7}$ lanthanide, or a mixed transition-metal/lanthanide ${ }^{8}$ strategy has yielded many structurally and magnetically interesting systems in recant years ${ }^{9}$. $o$-Vanillin as multidentate ligand displays a variety of bonding geometries, such as chelating, monodentate/bidentate bridging and chelating bridging ${ }^{1,10}$. Therefore, methods based on such bridging ligands (e.g., methoxide, hydroxide, alkoxide, oxide, carboxylate) have led to the formation of some interesting complexes ${ }^{11}$.

From the structural point of view, we selected the multidentate Schiff ligand, N-[2-(2-hydroxy-3-methoxybenzylideneamino)cyclohexyl]-2-hydroxy-benzamide $\left(\mathrm{H}_{2} L\right)$ formed by $o$-vanillin and $\mathrm{N}$-(2-aminocyclohexyl)-2-hydroxybenzamide, to construct coordination complexes. The Schiff base ligand $\left(\mathrm{H}_{2} L\right)$ contains adequate number of oxygen and nitrogen donors and these donors with suitable relative positions can chelate one or more metal centers. Accordingly, the design of such ligands is highly effective for establishing simple syntheses for new metal-assembled complexes ${ }^{12}$. In this contribution, we describe the synthesis and crystal structures of two mononuclear cobalt(III) and copper(II) complexes with $\mathrm{N}$-[2-(2-hydroxy-3-methoxybenzylideneamino)cyclohexyl]-2- hydroxy-benzamide $\left(\mathrm{H}_{2} L\right)$ ligand, $\left[\mathrm{Co}(\mathrm{H} L)_{2}\left(\mathrm{C}_{8} \mathrm{H}_{7} \mathrm{O}_{3}\right)\right] \cdot\left(\mathrm{CH}_{3} \mathrm{OH}\right)_{2}$ (1) and $\left[\mathrm{Cu}(\mathrm{H} L)_{2}\right](\mathbf{2})$.

\section{EXPERIMENTAL}

All the reagents and solvents employed were commercially available and used as received without further purification. Elemental analysis for $\mathrm{C}, \mathrm{N}$ and $\mathrm{H}$ were performed with a Perkin-Elmer 2400 II analyzer.

Preparation of $\mathbf{H}_{2} \boldsymbol{L}$ : N-(2-Aminocyclohexyl)-2-hydroxybenzamide was synthesized by the condensation of the 1,2diaminocyclohexane $(22.9 \mathrm{~g}, 0.2 \mathrm{~mol})$ and methyl 2-hydroxybenzoate $(15.2 \mathrm{~g}, 0.1 \mathrm{~mol})$ stirring at $80-100{ }^{\circ} \mathrm{C}$ for $13 \mathrm{~h}$ as reported method ${ }^{13}$. Then the white powders were collected by filtration, washed with cooled methanol and dried at room temperature. Yield: $10.9 \mathrm{~g}, 28.6 \%$. Elemental analysis (\%) calcd. for $\mathrm{C}_{13} \mathrm{H}_{18} \mathrm{~N}_{2} \mathrm{O}_{2}$ : C, 66.64, H, 7.74, N, 11.96; found: C, 67.23, H, 7.21, N, 12.06.

The ligand $\left(\mathrm{H}_{2} L=\mathrm{N}\right.$-[2-(2-Hydroxy-3-methoxybenzy-lideneamino)cyclohexyl]-2-hydroxybenzamide), was prepared in a similar manner to the reported procedures ${ }^{14,15}$. A methanol $(20 \mathrm{~mL})$ solution of $o$-vanillin $(0.1 \mathrm{~mol})$ was drop-wise added to a methanol solution $(20 \mathrm{~mL})$ of $\mathrm{N}$-(2-aminocyclohexyl)-2hydroxybenzamide $(0.1 \mathrm{~mol})$ and the mixture was stirred for $6 \mathrm{~h}$. Then the yellow precipitates of the $\mathrm{H}_{2} L$ ligand was separated and filtered off, washed with $5 \mathrm{~mL}$ of cooled methanol and then dried in air. Yield was $65 \%$. Elemental analysis (\%) calcd. for $\mathrm{C}_{21} \mathrm{H}_{24} \mathrm{~N}_{2} \mathrm{O}_{4}$ : C, 68.46; $\mathrm{H}, 6.57 ; \mathrm{N}, 7.60$; found $\mathrm{C}$, 68.71; H, 6.26; N, 7.52.

Preparation of complex 1: One pot method was used to synthesize complex 1, in which the Schiff base formed by the 
in situ condensation of $\mathrm{N}$-(2-aminocyclohexyl)-2-hydroxybenzamide $(0.1 \mathrm{mmol})$ and $o$-vanillin $(0.2 \mathrm{mmol})$ was utilized directly to react with $\mathrm{CoCl}_{2} \cdot 6 \mathrm{H}_{2} \mathrm{O}(0.1 \mathrm{mmol})$ in the presence of $\mathrm{NEt}_{3}(0.3 \mathrm{mmol})$ in $20 \mathrm{~mL}$ of methanol solution. The resulting mixture was stirred for $3 \mathrm{~h}$ at room temperature to afford brown solution, which was left unperturbed to allow the slow evaporation of the solvent. Black single crystals suitable for X-ray diffraction analysis were formed after about two weeks. Yield was $29.7 \%$ (based on Co). Elemental analysis (\%) calcd. for $\mathrm{C}_{52} \mathrm{H}_{61} \mathrm{~N}_{4} \mathrm{O}_{13} \mathrm{Co}$ : C, 61.63; H, 6.01; N, 5.48; found $\mathrm{C}, 61.89$; $\mathrm{H}, 6.09$; N, 5.55 .

Preparation of complex 2: Complex 2 was obtained by adding $\mathrm{Cu}\left(\mathrm{NO}_{3}\right)_{2} \cdot 3 \mathrm{H}_{2} \mathrm{O}(0.2 \mathrm{mmol})$ to a solution of $\mathrm{H}_{2} L(0.2$ $\mathrm{mmol}$ ) in $20 \mathrm{~mL}$ of methanol and the resulting mixture was stirred for about $3 \mathrm{~h}$ to afford a green solution. Two week later, green crystals of the title compound were isolated from the solution. Yield was $30.1 \%$ (based on $\mathrm{Cu}$ ). Elemental analysis (\%) calcd. for $\mathrm{C}_{42} \mathrm{H}_{46} \mathrm{~N}_{4} \mathrm{O}_{8} \mathrm{Cu}: \mathrm{C}, 63.18 ; \mathrm{H}, 5.81 ; \mathrm{N}, 7.02$; found C, 63.34; H, 5.56; N, 7.04.

\begin{tabular}{lll}
\multicolumn{3}{c}{ TABLE-1 } \\
\multicolumn{3}{c}{ CRYSTALLOGRAPHIC DATA AND STRUCTURE } \\
REFINEMENT FOR COMPLEXES 1 AND 2
\end{tabular}

X-Ray crystallography: Crystallographic data were collected on a Bruker Apex II CCD diffractometer with graphite monochromated $\mathrm{MoK}_{\alpha}$ radiation $(\lambda=0.71073 \AA)$ at a temperature of $185( \pm 2) \mathrm{K}$, for $\mathbf{1}$ and $\mathbf{2}$. Data processing was accomplished with the SAINT processing program. These structures were solved by the direct methods and refined on $\mathrm{F}^{2}$ by fullmatrix least squares using SHELXTL97 ${ }^{16,17}$. The location of $\mathrm{Co} / \mathrm{Cu}$ atom was easily determined and $\mathrm{O}, \mathrm{N}$ and $\mathrm{C}$ atoms were subsequently determined from the difference Fourier maps. All non-hydrogen atoms were refined with anisotropic thermal parameters. Crystallographic and refinement details of $\mathbf{1}$ and $\mathbf{2}$ are listed in Table-1. CCDC- 915373 (1) and 915374 (2) contain the supplementary crystallographic data for this paper. These data can be obtained free of charge from the Cambridge Crystallographic Data Centre via www.ccdc.cam.ac.uk/data_request/cif. [or from the Cambridge Crystallographic Data Centre (CCDC), 12 Union Road, Cambridge CB2 1EZ, UK; fax: (44) 1223-336033; email: deposit@ccdc.ccam.ac.uk].

\section{RESULTS AND DISCUSSION}

The molecular structure of $\mathbf{1}$ is displayed in Fig. 1 and all the relevant bond lengths and bond angles are listed in Table-2. The complex 1, $\left[\mathrm{Co}(\mathrm{HL})_{2}\left(\mathrm{C}_{8} \mathrm{H}_{7} \mathrm{O}_{3}\right)\right] \cdot\left(\mathrm{CH}_{3} \mathrm{OH}\right)_{2}$, contains of two $\mathrm{HL}^{-}$ligand, one $\mathrm{Co}(\mathrm{III})$ ion, one $o$-vanillin anion and two methanol molecules. The geometry around the $\mathrm{Co}(\mathrm{III})$ ion centre can be described as a distorted octahedral geometry composed of the $\mathrm{N}_{2} \mathrm{O}_{4}$ set of atoms, the $\mathrm{HL}^{-}$ligand is chelated to the $\mathrm{Co}(\mathrm{III})$ ion in an $\mathrm{O}, \mathrm{N}$-bidentate manner and the $o$-vanillin anion coordinates through the phenolate oxygen atom and aldehyde oxygen. The Co1-O and Co1-N distances are similar to the corresponding distances observed in some similar Co

\begin{tabular}{llll}
\hline \multicolumn{5}{c}{ TABLE-2 } \\
\multicolumn{5}{c}{ BOND LENGTHS $(\AA)$ AND ANGLES $\left({ }^{\circ}\right)$ FOR COMPLEX 1} \\
\hline Bond lengths & & \\
\hline Co1-O1 & $1.897(3)$ & Co1-N1 & $1.955(4)$ \\
Co1-O3 & $1.903(4)$ & Co1-N4 & $1.951(3)$ \\
Co1-O8 & $1.880(3)$ & Co1-O9 & $1.893(3)$ \\
\hline Bond angles & & & \\
\hline O8-Co1-O9 & $88.03(12)$ & O8-Co1-O3 & $94.46(15)$ \\
O8- Co1-O1 & $85.92(12)$ & O9-Co1-O3 & $87.23(14)$ \\
O9- Co1-O1 & $171.95(13)$ & O1-Co1-O3 & $87.97(14)$ \\
O8- Co1-N4 & $176.68(15)$ & O9-Co1-N4 & $92.19(13)$ \\
O1- Co1-N4 & $94.16(13)$ & O3-Co1-N4 & $88.86(16)$ \\
O8- Co1-N1 & $84.42(14)$ & O3-Co1-N1 & $94.01(14)$ \\
O1- Co1-N1 & $90.67(14)$ & O3-Co1-N1 & $178.30(14)$ \\
N4- Co1-N1 & $92.26(15)$ & & \\
\hline
\end{tabular}

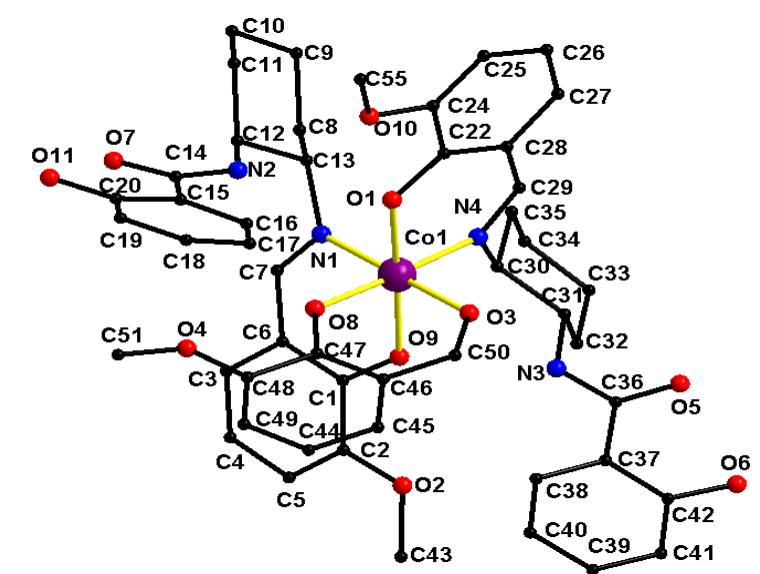

Fig. 1. A view of the complex 1, showing the atomic numbering scheme, methanol molecules are omitted for clarity 
complexes $^{18,19}$. The structure shows distortion from perfect octahedral geometry with respect to bond angles. The trans base angles $\left[\mathrm{N} 1-\mathrm{Co} 1-\mathrm{O} 3=178.30(14)^{\circ}, \mathrm{N} 4-\mathrm{Co} 1-\mathrm{O} 8=176.68\right.$ $\left.(15)^{\circ}\right]$ are all significantly less than the ideal value of $180^{\circ}$. The axial angle $\mathrm{O} 1-\mathrm{Co} 1-\mathrm{O} 9\left[171.95(13)^{\circ}\right]$ deviates significantly from the ideal $180^{\circ}$. This angle of distortion can be attributed to the ligand structure.

The present complex 2 of $\left[\mathrm{Cu}(\mathrm{H} L)_{2}\right]$, together with the molecular structure is displayed in Fig. 2. Selected bond lengths and angles are given in Table-3. The title complex $\mathbf{2}$ contains of two $\mathrm{H} L^{-}$ligand and one $\mathrm{Cu}$ (II) ion. The geometry around the $\mathrm{Cu}$ (II) centre can be described as a distorted square-planar composed of the ${ }_{\mathrm{N} 2} \mathrm{O}_{2}$ set of atoms, every $\mathrm{H}^{-}$ligand is chelated to the $\mathrm{Cu}$ atom in an $\mathrm{O}, \mathrm{N}$-bidentate manner. The $\mathrm{Cu} 1-\mathrm{O}$ and $\mathrm{Cu} 1-\mathrm{N}$ distances are similar to the corresponding distances observed in some similar $\mathrm{Cu}$ complexes ${ }^{20-22}$. There is slightly deviation of the metal centre from the $\mathrm{N}_{2} \mathrm{O}_{2}$ equatorial plane. The maximum displacements from the least-squares plane through N2, N4, O4 and O8 are 0.5447 (22) $\AA$ and 0.3598 (18) $\AA$ for atoms N4 and O8, respectively, Cu1 is 0.1208 (2) $\AA$ below this plane.

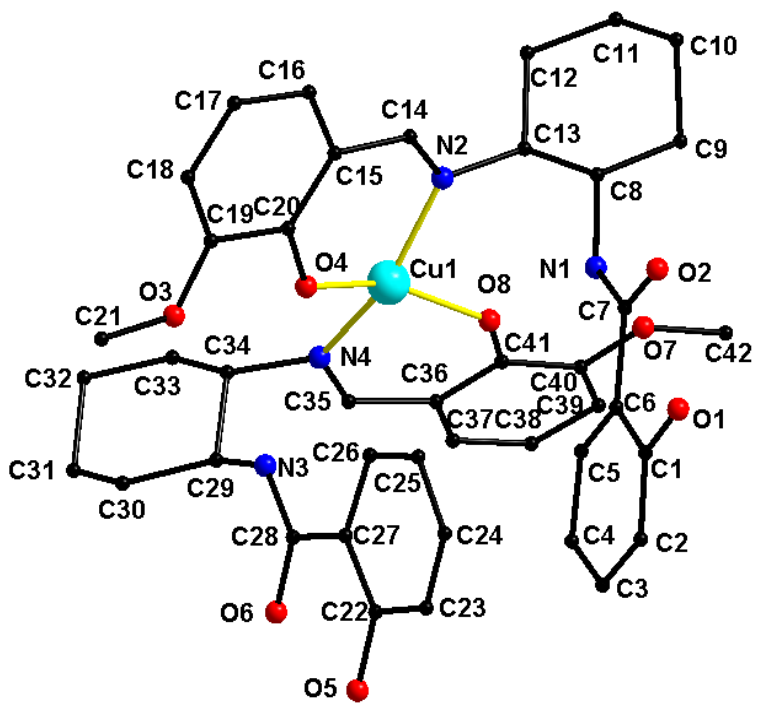

Fig. 2. A view of the complex 2, showing the atomic numbering scheme

TABLE-3

BOND LENGTHS $(\AA)$ AND ANGLES $\left(^{\circ}\right)$ FOR COMPLEX 2

\begin{tabular}{llll}
\hline Bond lengths & & & \\
\hline Cu1-O8 & $1.886(6)$ & Cu1-N2 & $1.969(7)$ \\
$\mathrm{Cu} 1-\mathrm{O} 4$ & $1.891(5)$ & $\mathrm{Cu} 1-\mathrm{N} 4$ & $1.998(7)$ \\
\hline Bond angles & & & \\
\hline O8-Cu1-O4 & $151.94(7)$ & O8-Cu1-N2 & $92.4(3)$ \\
O4-Cu1-N2 & $92.3(3)$ & O8-Cu1-N4 & $93.2(3)$ \\
O4-Cu1-N4 & $91.2(3)$ & N2-Cu1-N4 & $91.2(3)$ \\
\hline
\end{tabular}

The ligand $\mathrm{H}_{2} L$ is a potential multidentate ligand with $\mathrm{N}_{2} \mathrm{O}_{4}$ donor sites but in this case it acts as a bidentate chelate. In complex $\mathbf{1}$ and complex $\mathbf{2}$, the $\mathrm{H} L^{-}$ligand is chelated to the metal ions only in an O,N-bidentate manner, which from the $o$-vanillin group. 2-Hydroxybenzamide group is not effected the coordination metal cation, thus the angle of both aromatic rings in each ligand is similarly. In complex $\mathbf{1}$, both aromatic rings in each ligand surrounding the metal ions form a dihedral angle that is $78.40(16)^{\circ}$. The angle is similar to complex 2 of $78.13(9)^{\circ}$.

\section{Conclusion}

Reactions of a multidentate N-[2-(2-Hydroxy-3-methoxybenzylideneamino)cyclohexyl]-2-hydroxy-benzamide $\left(\mathrm{H}_{2} L\right)$ with $\mathrm{CoCl}_{2} \cdot 6 \mathrm{H}_{2} \mathrm{O}$ and $\mathrm{Cu}\left(\mathrm{NO}_{3}\right)_{2} \cdot 3 \mathrm{H}_{2} \mathrm{O}$ in methanol solvent results in the formation of two mononuclear complexes, $\left[\mathrm{Co}(\mathrm{HL})_{2}\left(\mathrm{C}_{8} \mathrm{H}_{7} \mathrm{O}_{3}\right)\right] \cdot\left(\mathrm{CH}_{3} \mathrm{OH}\right)_{2}(\mathbf{1})$ and $\left[\mathrm{Cu}(\mathrm{HL})_{2}\right](\mathbf{2})$. Both complexes are characterized by elemental analysis and crystal structure analysis. The metal centers of complexes $\mathbf{1}$ and $\mathbf{2}$ have exhibit a distorted octahedral geometry and squareplanar geometry, respectively. The multidentate $\mathrm{H} L^{-}$ligand is chelated to the metal ions only in an O,N-bidentate manner, which from the $o$-vanillin group, thus it is a pity that no synthetic polynuclear metal complexes.

\section{ACKNOWLEDGEMENTS}

The authors thank the project supported by the Development and Reform Commission of Jilin Province, the Science and Technology Burrau of Changchun City, the Science and Technology Department of Jilin Province, China (2011174) and Changchun University of Science and Technology for their financial support.

\section{REFERENCES}

1. S.H. Li, S.K. Gao, S.X. Liu and Y.N. Guo, Cryst. Growth Des., 10, 495 (2010).

2. H. Yin and S.X. Liu, J. Mol. Struct., 918, 165 (2009).

3. L.F. Zou., L. Zhao, Y.N. Guo, J.K. Tang, Q.L. Wang and Y.H. Li, Inorg. Chim. Acta, 382, 65 (2012).

4. J.P. Costes, F. Dahan and F. Nicodeme, Inorg. Chem., 40, 5285 (2001).

5. J. Tang, I. Hewitt, N.T. Madhu, G. Chastanet, W. Wernsdorfer, C.E. Anson, C. Benelli, R. Sessoli and A.K. Powell, Angew. Chem. Int. Ed., 45, 1729 (2006).

6. Y. Elerman, A. Elmali and I. Svoboda, Z Naturforsch B, 57, 519 (2002).

7. C.D. Papadopoulos, A.G. Hatzidimitriou, G.P. Voutsas and M. LaliaKantouri, Polyhedron, 26, 1077 (2007).

8. T. Hamamatsu, K. Yabe, M. Towatari, N. Matsumoto, N. Re, A. Pochaba and J. Mrozinski, Bull. Chem. Soc. Jpn., 80, 523 (2007).

9. G.M. Yu, L. Zhao, L.F. Zou, Y.N. Guo, G.F. Xu, Y.H. Li and J.K. Tang, J. Chem. Crystallogr., 46, 606 (2011).

10. E.A. Velcheva, B.A. Stamboliyska and P.J. Boyadjieva, J. Mol. Struct., 963, 57 (2010).

11. L.K. Thompson, C.J. Matthews, L. Zhao, Z.Q. Xu, D.O. Miller, C. Wilson, M.A. Leech, J.A.K. Howard, S.L. Heath, A.G. Whittaker and R.E.P. Winpenny, J. Solid State Chem., 159, 308 (2001).

12. M. Koikawa, H. Yamashita and T. Tokii, Inorg. Chim. Acta, 357, 2635 (2004).

13. R. Mitsuhashi, T. Suzuki, Y. Sunatsuki and M. Kojima, Chem. Lett., 40, 696698 (2011).

14. Y. Qiao, X.P. Ju, Z.Q. Gao and L.Q. Kong, Acta Cryst. E, 66, O95 (2009).

15. O. Pouralimardan, A.-C. Chamayou, C. Janiak and H. HosseiniMonfared, Inorg. Chim. Acta, 360, 1599 (2007).

16. G.M. Sheldrick, SHELXS-97, Program for Crystal Structure Solution, University of Göttingen, Germany (1997).

17. G.M. Sheldrick, SHELXL-97, Program for Crystal Structure Refinement, University of Göttingen, Germany (1997).

18. M. Fleck, D. Karmakar, M. Ghosh, A. Ghosh, R. Saha and D. Bandyopadhyay, Polyhedron, 34, 157 (2012).

19. A. Ray, S. Banerjee, G.M. Rosair, V. Gramlich and S. Mitra, Struct. Chem., 19, 459 (2008).

20. H. Ali, N.A. Khamis, W.J. Basirun and B.M. Yamin, Acta Cryst. E, 60, m982 (2004).

21. A. Elmali, Y. Elerman and I. Svoboda, Acta Cryst., C56, 423 (2000).

22. L.F. Zou, X.Y. Yang, Y. Gao, H.B. Yao and Y.H. Li, Acta Cryst. E, 67, m511 (2011). 